\title{
Minimum Interference Resource Allocation Algorithm for D2D in C-RAN Architecture
}

\author{
${ }^{1}$ Hou Weina, ${ }^{2}$ Yu Zhonghua*, ${ }^{3}$ Chen Linglong, ${ }^{4}$ Liu Zhanjun \\ ${ }^{1,2,3,4}$ Chongqing Key Laboratory of Mobile Communications Technology, \\ Chongqing University of Posts and Telecommunications, Chongqing 400065 \\ ChinaHouwn@cuqpt.edu.com,Timer_yu@163.com,chen_89_11@163.com, \\ liuzj@cqupt.edu.com
}

\begin{abstract}
In order to solve the problem of supporting resource allocation of D2D in C-RAN architecture, Minimum Interference Resource Allocation (MIRA) is proposed by using the centralized processing of C-RAN to obtain the mutual interferences among users. The proposed scheme takes the minimum aggregation of mutual interferences among users as the target to construct the optimization mathematical model, and then to achieve the optimal solution. The simulation results show that even though the SINR of D2D users is decreased to some extent by adopting the proposed MIRA algorithm, the performance of network throughput, the number of connections and the SINR of cellular users are improved efficiently compared with other schemes.
\end{abstract}

Keywords: C-RAN; D2D users; centralized processing; optimization; resource allocation

\section{Introduction}

C-RAN architecture supports collaborative communications, energy conservation, centralized processing, etc. [1-4], and can achieve D2D communication more easily.

Our previous work proposes a D2D communication mechanism in C-RAN architecture with the characteristics of achieving high data rate, low latency and low power consumption, and the research work was published as reference [5]. However, the lack of correspondingly resource allocated mechanism causes significant interference between users.

The current resource allocation of D2D mainly focused on the LTE network [6-8], studying for resource allocation problems about multi-group D2D users reusing the same spectrum resources of the same cellular subscriber. In [6], a resource allocation algorithm based on cooperative game is proposed, in [8] a semi-distributed resource allocation algorithm is designed. Only intra-cell interference is considered in the single-cell resource allocation algorithm, but the inter-cell interference is ignored. The resource allocation mechanism in [9] using the Hungarian algorithm to achieve inter cell interference coordination, but the mutual interference between D2D users and cellular subscribers is not considered. And the characteristic of centralized processing in C-RAN architecture is not used adequately. So the study of resource allocation algorithm which D2D users exist is meaningful to improve network performance in C-RAN architecture.

\section{System Model}

We assume the network of C-RAN architecture consisting of $K$ cells, and each one share $M$ resources; the number of cellular users and D2D users in each cell is $\boldsymbol{C}=\left\{c_{1}, \cdots c_{k}, \cdots c_{K}\right\}$ and $\boldsymbol{D}=\left\{d_{1}, \cdots d_{k}, \cdots d_{K}\right\}$, respectively, the number of users per 
cell can be expressed as:

$$
n_{k}=c_{k}+d_{k}
$$

$P_{c} 、 P_{d}$ is the transmitted power of cellular users and D2D users respectively. So the received signal of cellular users $c, \quad c \in C$ on the $m$ th RB, $m \in M$ can be expressed as:

$$
y_{c, m}=\sqrt{p} g_{c, m} x_{c, m}+\sum_{u \in \boldsymbol{I}_{c}} \sqrt{P_{u}} g_{u, m} x_{u, m}+w_{c, m}
$$

$g_{c, m}$ is the channel gain of user $c$ achieved on the $m$ th RB.

$\boldsymbol{I}_{\boldsymbol{c}}$ represents interference user set that interfere with cellular users $C$.

$x_{c, m}$ is transmitted data expressed by users $\boldsymbol{C}$ on resource block $m$.

$w_{c, m}$ represents additive white gaussian noise, with the power spectral density $N_{0}$.

$P_{u}$ is transmitted power expressed by user $u$ who is the interfered user of $C$, and satisfy the following:

$$
P_{u}= \begin{cases}P_{c} & u \in C \\ P_{d} & u \in D\end{cases}
$$

The SINR of user $c$ on resource block $m$ is:

$$
\gamma_{c, m}=\frac{P_{c} g_{c, m}}{\sum_{u \in I_{c}} P_{u} g_{u, m}+N_{0}}
$$

The received signal of $\mathrm{D} 2 \mathrm{D}$ user $d, d \in D$ on the $m$ th RB can be expressed as:

$$
y_{d, m}=\sqrt{p}_{d} g_{d, m} x_{d, m}+\sum_{u \in I_{d}} \sqrt{P_{u}} g_{u, m} x_{u, m}+w_{d, m}
$$

The SINR of user $d$ on resource block $m$ is:

$$
\gamma_{d, m}=\frac{P_{d} g_{d, m}}{\sum_{u \in I_{d}} P_{u} g_{u, m}+N_{0}}
$$

According to Shannon capacity formula, the $R_{m}$ of cellular subscribers, D2D users can obtain respectively is the following:

$$
\begin{aligned}
& R_{c}=\sum_{m=1}^{M} a_{c, m} \log \left(1+\gamma_{c, m}\right) \\
& R_{d}=\sum_{m=1}^{M} a_{d, m} \log \left(1+\gamma_{d, m}\right)
\end{aligned}
$$

which $a_{c, m} 、 a_{d, m} \in \boldsymbol{A}, \boldsymbol{A}=\left[\boldsymbol{A}_{1}, \boldsymbol{A}_{2}, \cdots, \boldsymbol{A}_{k}, \cdots, \boldsymbol{A}_{K}\right]$,

wherein $\boldsymbol{A}_{\boldsymbol{k}}=\left[\begin{array}{ccc}a_{1,1}^{k}, \cdots, a_{1, c_{k}}^{k}, \cdots, a_{1, n_{k}}^{k} \\ \vdots & \vdots & \vdots \\ a_{M, 1}^{k}, \cdots, & a_{M, c_{k}}^{k}, \cdots, a_{M, n_{k}}^{k}\end{array}\right]$ is the allocation vector of system RB.

$a_{m, n_{k}}$ is binary variable, $a_{m, n_{k}}=1$ presents the $n_{k}$ th user in cell $k$ using resource block $m$.

The sum of system interference is:

$$
I_{s}=\sum_{k=1}^{K} \sum_{n=1}^{n_{k}} \sum_{m=1}^{M} a_{m, n}^{k} p_{n}^{k} g_{n, u}^{k}
$$


Here $u \in I, \quad I=I_{c} \cup I_{d}$.

In order to avoid the same frequency interference in a cell, each RB can be used only by a cellular user or a D2D user. Resource block should be allocated to the cellular users for uplink and downlink. However, it needs RB which transmits directly for the D2D users and each link can get only one RB. The optimization model for resource allocation of cellular users, D2D users coexisting in C-RAN architecture is expressed by:

$$
\begin{gathered}
I_{s}=\min \sum_{k=1}^{K} \sum_{n=1}^{n_{k}} \sum_{m=1}^{M} a_{m, n}^{k} p_{n}^{k} g_{n, u}^{k} \\
\text { s.t }\left\{\begin{array}{l}
\sum_{n=1}^{n_{k}} a_{m, n}^{k} \leq 1 \\
\sum_{m=1}^{M} a_{m, n}^{k} \leq 1 \\
2 * c_{k}+d_{k} \leq M
\end{array}\right.
\end{gathered}
$$

The first formula in restrictive condition means that resource block $m$ can only be used by a cell user or a D2D user, the second formula ensures that only one resource block can be used by user $n$, the third formula guarantees that the number of accessed users should be less than or equal to the number of resource block.

\section{Resource Allocation Mechanism}

Based on the mathematical model above, the Minimum Interference Resource Allocation mechanism (MIRA: Minimum Interference Resource Allocation) by using the idea of iterative solution is proposed as Figure1. 


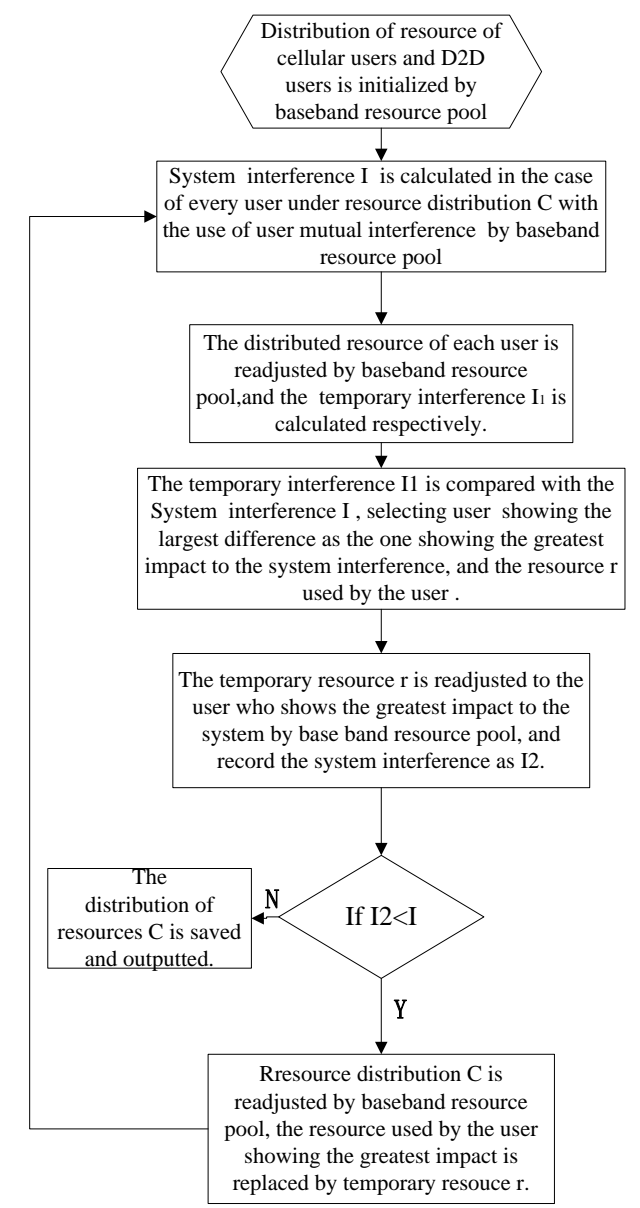

Figure 1. Flow Chart of Resource Allocation of MIRA Algorithm

The steps of MIRA algorithm is as the following.

Step-1.The resource of users is allocated by base band resource pool, the initial resource allocation is saved as resource allocation $C$.

Step-2. System interference $I$ is calculated according to the mutual user interference database which is achieved by the base band resource pool by the interference coordination scheme.

Step-3. Temporary system interference $I_{1}$ is calculated when every user takes turn to use other resource in base band resource pool.

Step-4. Compared $I_{1}$ with $I$, select user showing the largest differences as the one showing the greatest impact to the system interference and calculate the temporary resource $r$ used by the users.

Step-5. The resource allocated to the user shows the greatest impact on the system is adjusted to R and the system interference $I_{2}$ is calculated, compare $I_{2}$ with $I$.

Step-6. If $I_{2}$ is less than $I$, the allocated resource $C$ is updated, then do Step2.Otherwise, keep the original base band resource pool distribution system $C$ unchanged, and using this resource allocation result to D2D users and cellular users to allocate resources. 


\section{Simulation and Performance Analysis}

\subsection{Simulation Environment}

To investigate the performance of MIRA, a simulation of C-RAN architecture is conducted with the help of MATLAB, and both cellular users and D2D users are in the system. The pass loss model of cellular users and D2D users can be expressed as $128.1+37.6 \log _{10}(d[\mathrm{~km}])$ and $148+40 \log _{10}(d[\mathrm{~km}])$ [10] respectively. The number of resource block is 14 and the system frequency reuse factor is 1 . The transmit power of cellular users is $24 \mathrm{dbm}$ and the maximum distance of D2D users is $40 \mathrm{~m}$. Meanwhile the noise power spectral density is $-174 \mathrm{dbm} / \mathrm{Hz}$ and infinite buffer is chosen as simulation model. Related simulation parameters is shown in Table 1 . The weights of the same frequency interference $W_{n}$ is $10^{5}$ [11], the interference outside the range and from non-adjacent cell $W_{z}$ is 0 . Effective interference in interference range is the signal intensity which received by the user who is disturbed.

Table 1. Simulation Parameters

\begin{tabular}{cc}
\hline Parameter & Values \\
\hline Number of cells & 19 \\
Cell radius of cells /m & 100 \\
Number of resource block & 14 \\
System frequency reuse factor & 1 \\
Transmit power of cellular users & 43 \\
/dbm $\quad$ Path loss of cellular users & $128.1+37.6 \log _{10}(d[\mathrm{~km}])$ \\
Transmit power of D2D users /dbm & 24 \\
Path loss of D2D users & $148+40 \log _{10}(\mathrm{~d}[\mathrm{~km}])$ \\
The maximum distance of D2D users & 40 \\
$/ \mathrm{m} \quad$ Noise power spectral density & -174 \\
$(\mathrm{dbm} / \mathrm{Hz})$ & Simulation model
\end{tabular}

The users whose distance of the nearest RRU is more than $20 \mathrm{~m}$ and the distance between the users less than $15 \mathrm{~m}$ will be chosen as a pair of D2D users. If there are more than one users can meet this condition, then randomly selected two users. In the simulation process, when the delivery process of RRU is used, the resource block is allocated to the uplink and downlink, which is the users to the RRU and the RRU to the users respectively, and the system throughput is calculated. When the direct communication is used, the resource block only allocated to the direct communication link, and the system throughput is calculated too. The number of the users that the system supported is the users who has been allocated with resource block. The physical resources of D2D users and RRU forwarding users used is mutually orthogonal, unified by a centralized processing baseband resource pool, and the smallest unit of scheduling is a RB.

In this simulation process, we selected that the recent RRU distance is more than $20 \mathrm{~m}$ and the distance between the users is less than $15 \mathrm{~m}$ of two users as a pair of D2D users. 
The RB scheduling rule is: when the number of users is smaller than the number of RB, the polling distribution is used until assignment of RB is completed; when the number of users in the system is greater than the RB number, the saving resource by using D2D will be allocated to the users without $\mathrm{RB}$, and the RB assigned the each user is no more than one.

\subsection{Performance Analysis}

Performance between MIRA and ARA on throughput, access number of users and SINR of the users of cellular users and D2D users in C-RAN architecture is compared as following.

1. Throughput

Figure 2 to Figure 4 shows the throughput CDF graph of using ARA and MIRA algorithm.

As it is shown in the Figure, Figure 2 is the throughput CDF graph of the system, Figure 3 is the throughput CDF graph of cellular users while Figure 4 is the throughput CDF graph of D2D users. It can be seen from the Figure, the throughput is improved by using MIRA more than ARA algorithm, the reason is that in MIRA, the resource saved by D2D users is allocated to other D2D users or cellular users, by doing this, more users can be accessed into the system, so the throughput of the system, the cellular users and D2D users is promoted.

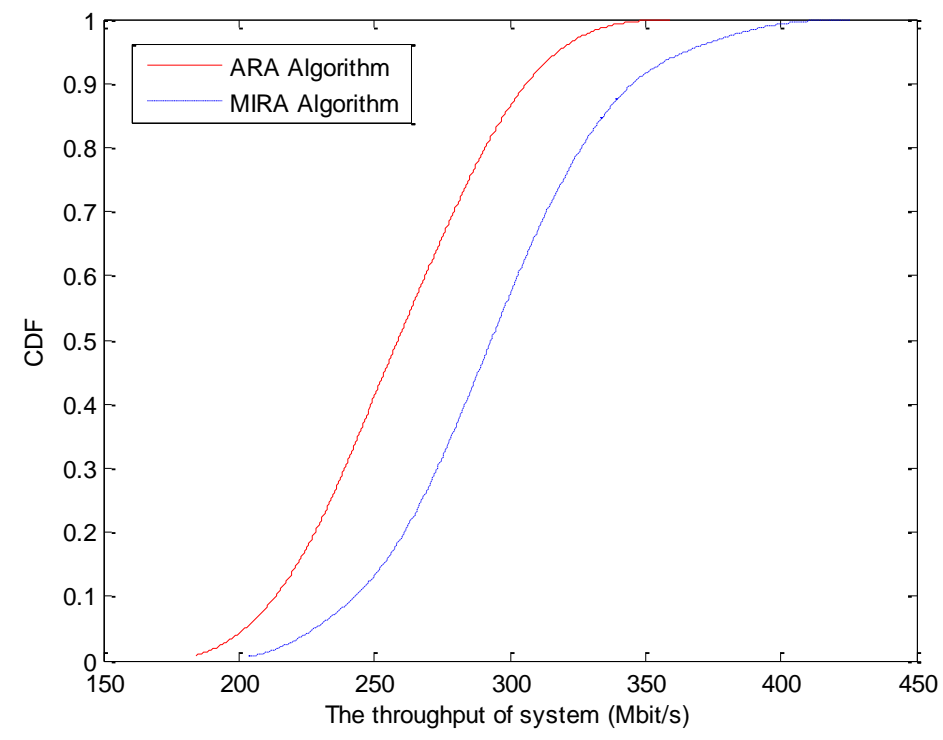

Figure 2. The Throughput of System 


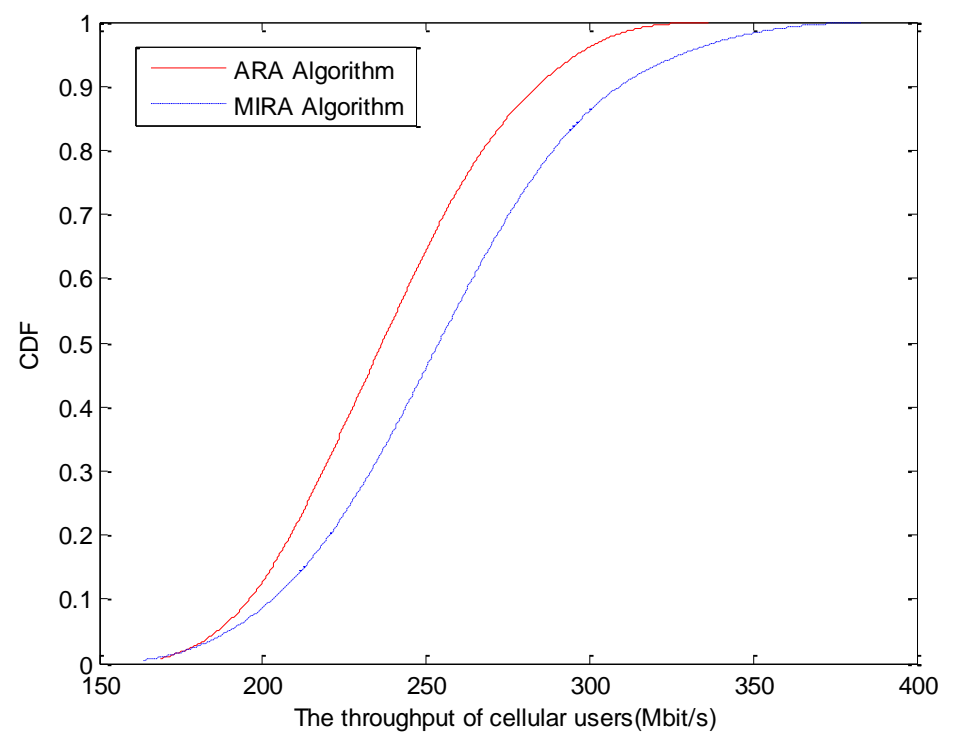

Figure 3. The Throughput of Cellular Users

2. Access number of users

Figure 5 is the CDF graph of number of accessed users with the use of ARA and MIRA algorithm.

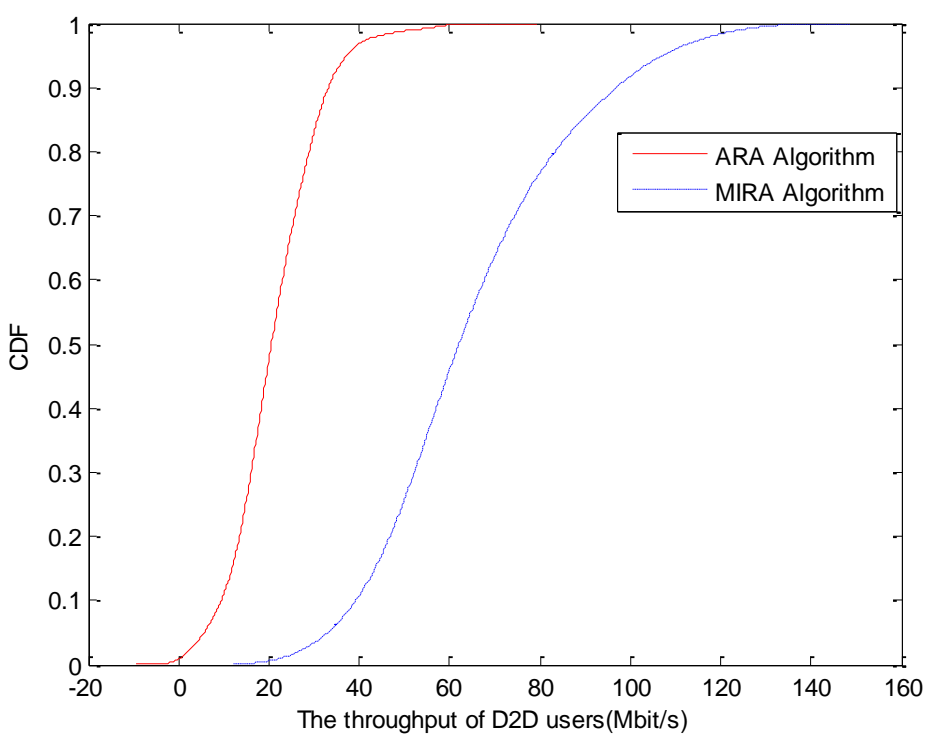

Figure 4. The Throughput of D2D Users 


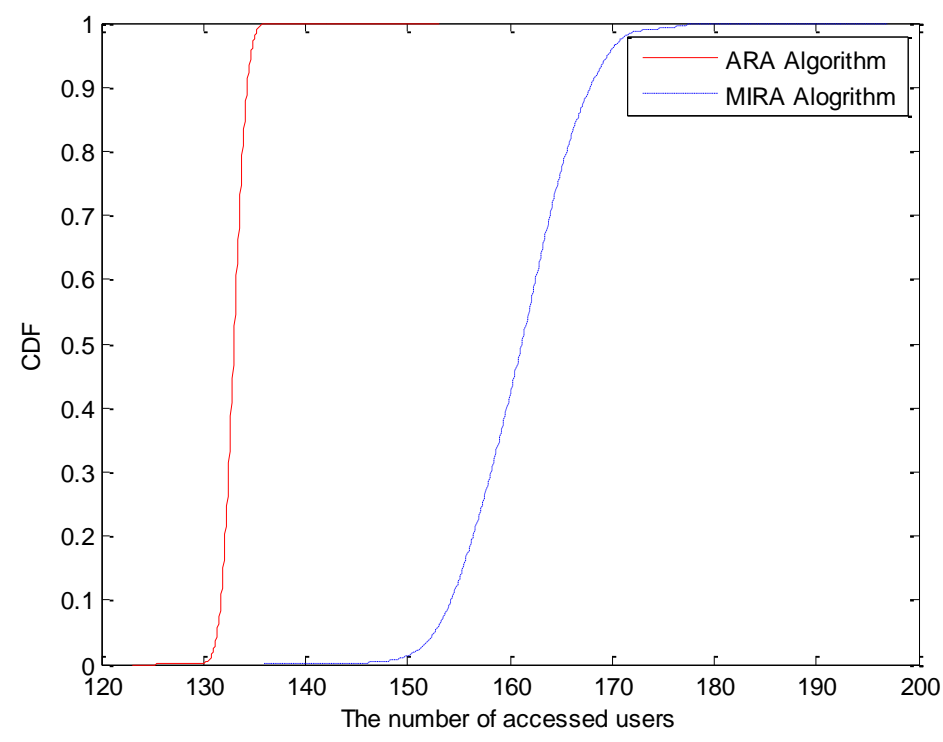

Figure 5. The Number of Accessed Users

As it is shown in the Figure, more users can be accessed into the system by using MIRA algorithm, this is achieved by allocating the resource which D2D users saved to the users without resource, so the number of accessed users is increased. At $50 \%$ of the CDF graph, the number of system users can be increased by 28 , the reason for this is that the resource saved by D2D users is allocated to users without resource, so more users can be accessed into the system by using MIRA algorithm.

3. Comparison of SINR of the users

Figure 6 to Figure 8 shows the SINR graph of the users using ARA and MIRA algorithm.

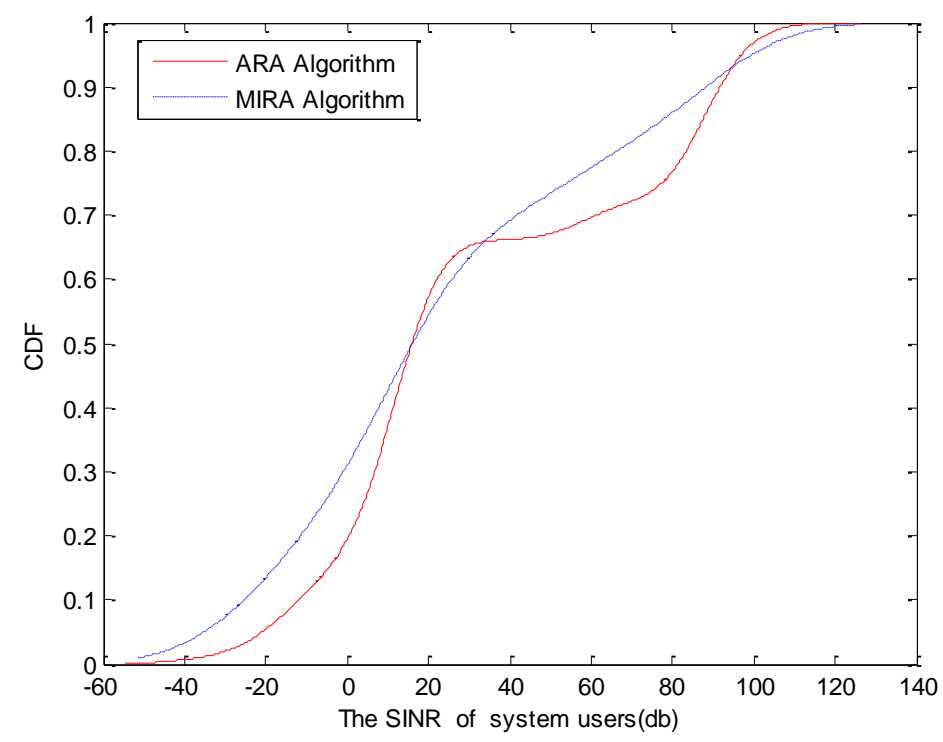

Figure 6. The SINR of System Users 


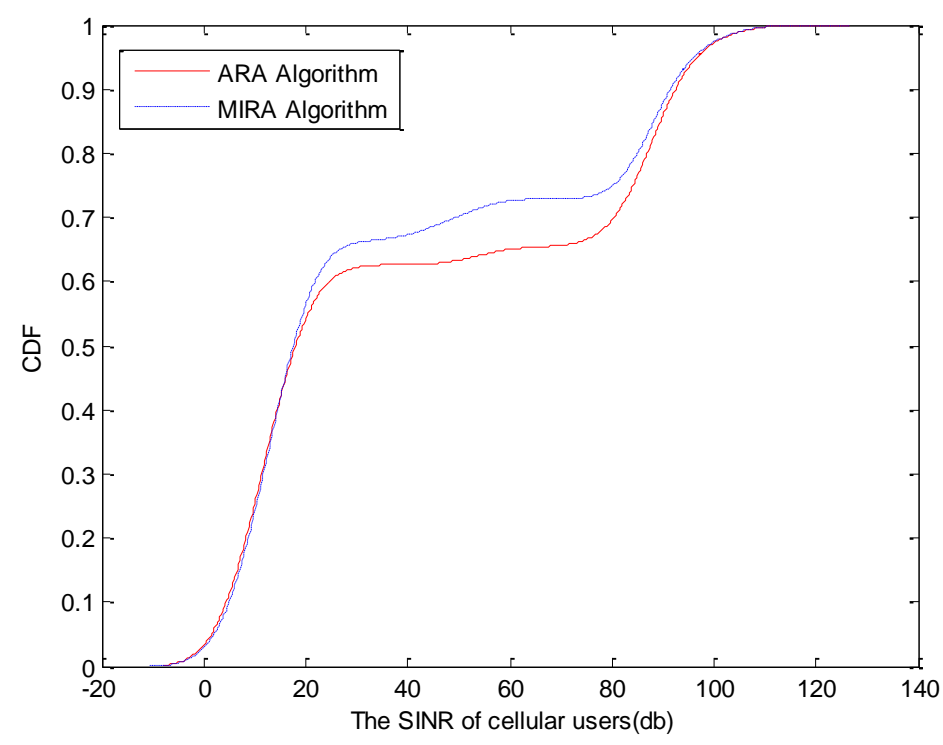

Figure 7. The SINR of Cellular Users

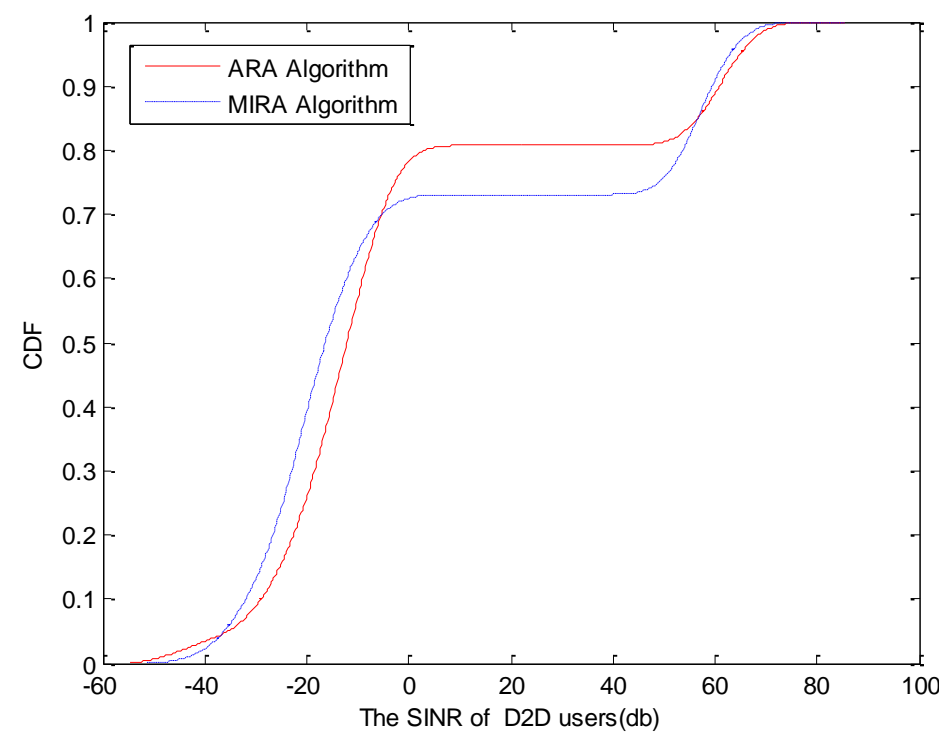

Figure 8. The SINR of D2D Users

It can be seen from Figure 6, compared with ARA algorithm, SINR of the system users is decreased by using MIRA algorithm; it shows in Figure 7 that the SINR of cellular users and D2D users is almost the same by using both of the algorithms, so the performance of cellular users is protected by using MIRA algorithm; Figure 8 shows that SINR of D2D users is poor, when the value of the vertical coordinate is 0.5 , the SINR improved by $4 \mathrm{db}$ when the ARA algorithm is used, the reason is that the interference increased with more cellular users and D2D users access into the system, meanwhile, as the interference range of and the transmitted power of D2D users smaller than cellular users, so the interference of D2D users is larger than cellular users.

It can be seen from Figure 6, compared with ARA algorithm, SINR of the system users is decreased by using MIRA algorithm; it shows in Figure 7 that the SINR of cellular users and D2D users is almost the same by using both of the algorithms, so the 
performance of cellular users is protected by using MIRA algorithm; Figure 8 shows that SINR of D2D users is poor, when the value of the vertical coordinate is 0.5 , the SINR improved by $4 \mathrm{db}$ when the ARA algorithm is used, the reason is that the interference increased with more cellular users and D2D users access into the system, meanwhile, as the interference range of and the transmitted power of D2D users smaller than cellular users, so the interference of D2D users is larger than cellular users.

1. Comparison of the SINR of the edge users

Figure 9 to Figure 11 shows the SINR graph of the edge users using ARA and MIRA algorithm.

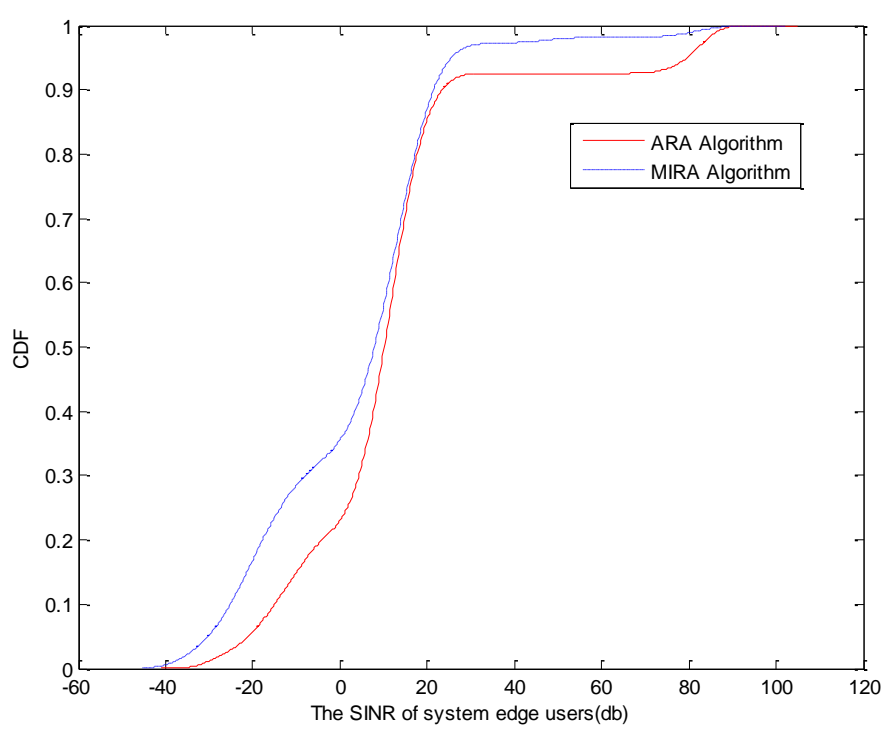

Figure 9. The SINR of System Edge Users

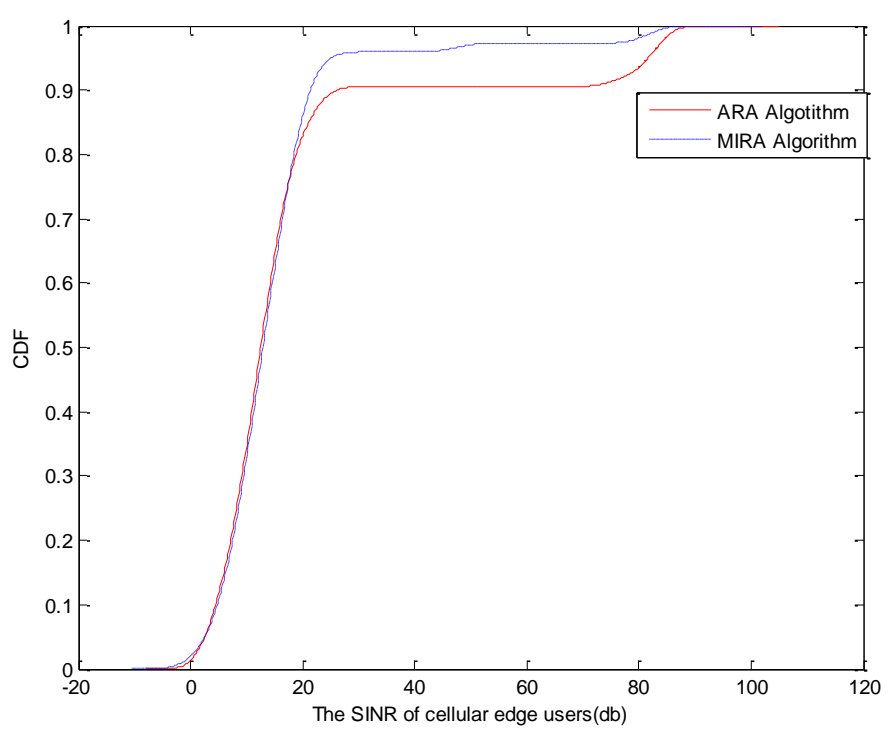

Figure 10. The SINR of Cellular Edge Users 


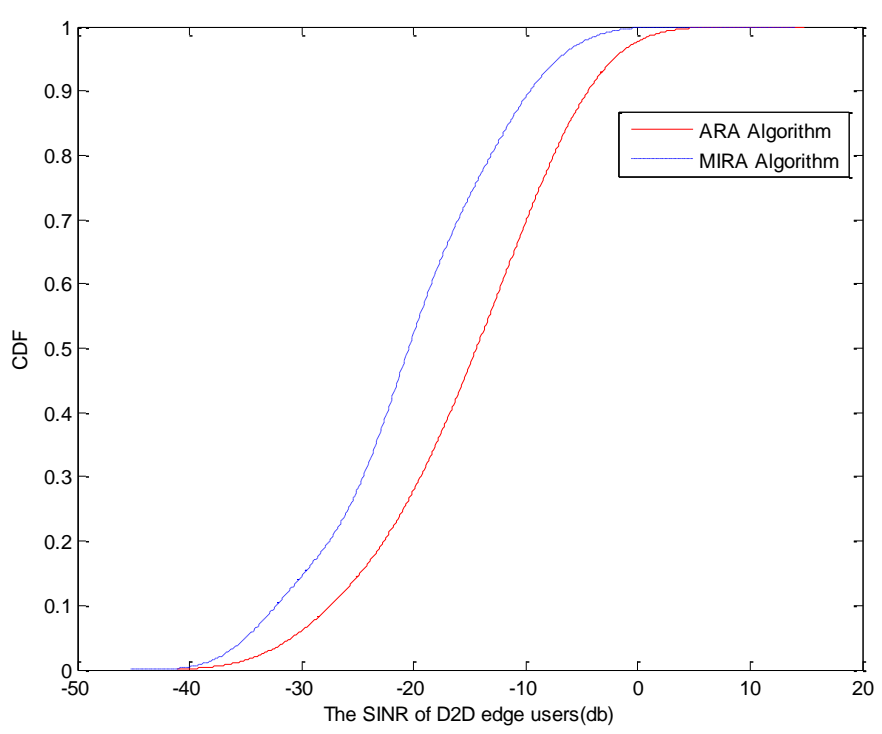

Figure 11. The SINR of D2D Edge Users

It can be seen from Figure 9, compared with ARA algorithm, SINR of the system edge users is decreased by using MIRA algorithm. From Figure 10, we can see that the SINR of the cellular edge users is almost the same by using ARA algorithm and MIRA algorithm. From Figure 11, it is showed that the SINR of D2D users is decreased about $8 \mathrm{db}$ when the value of vertical coordinates is 0.5 . This is mainly because when the MIRA algorithm is used, the number of the accessed users is increased, the interference of cell edge users is stronger, compared with the interference of cell center users, at the same time, the D2D users are limited to the transmitting power and the smaller interference range, so the interference of the D2D edge users is more obviously.

From Figure 6 to Figure 11, it can be seen that the SINR of cell users is the same by using MIRA and ARA algorithm, at the same time, the SINR of D2D users decreased by a little. When combined with Figure 2 to Figure 4, it can be found that more throughput can be achieved when MIRA algorithm is used. Meanwhile, Figure 5 shows that more users can be accessed to the system by using MIRA algorithm. In this paper, the MIRA algorithm can obtain higher system throughput, the number of accessed users and guarantee the cellular user SINR by sacrificing the D2D user's SINR. The MIRA algorithm makes full use of the same frequency interference between cellular users and D2D users by the centralized management of C-RAN networks.

\section{Conclusion}

The problem of resource allocation in C-RAN Architecture is translated into interference management between users, i.e. on the base of interference between multicell D2D users and cellular users, the optimization function which aimed at making the interference be minimum is constructed, so the interference management is transformed into optimization problem, the resource allocated scheme is the optimization solution of this problem.

According to the resource allocation mechanism of direct communication in C-RAN architecture, by using effective interference of cellular users and D2D users which is predefined, the interference between users is calculated, the interference date base between users is constructed; the interference of system is calculated by using interference date base, so the optimization function aimed at the interference in system is established; the resource allocation scheme of D2D users and cellular users is obtained by the iterative 
computation of the system interference which can achieve this result that is convergent resource allocation.

Simulation results show that at the expense of SINR of D2D users, the SINR of cellular users is guaranteed, more users can be accessed into the system and a higher throughput is achieved by using MIRA algorithm. In this algorithm, the centralized management of CRAN architecture is fully used to coordinate the co-frequency interference between cellular users and D2D users.

The deficiency of this paper is that the resource sharing between D2D users and cellular users in the same cell is not considered. The MIRA algorithm uses orthogonal resource allocation by the users in the same cell to avoid co-channel interference between users, but it is not conducive to the system to improve the resource utilization. While D2D cell users share the same resources with the cellular users, the cellular users will not only be interfered by the cellular user from the adjacent cell, but also from the D2D users in the same cell, and at the same time, the interference of the D2D users will be changed too. In multi cell, by the way of shared resources with D2D users and cellular users in the same cell can significantly increase network capacity and resource utilization. However, the interference problem will be more severe, therefore at the same time, relevant research in this area is very significant.

\section{Acknowledgements}

This work is supported by National 863 Project (2014AA01A701).

\section{References}

[1] J. Qiao, X. Shen, J. J. W. Mark, Q. Shen, Y. He and L. Lei, "Enabling Device-to-Device Communications in Millimeter-Wave 5G Cellular Networks", IEEE Communications Magazine, vol. 1, no. 53, (2015)

[2] IMT-2020 (5G) promotion team. White Paper -5G vision and demand [EB/OL] Beijing: IMT-2020 (5G) promotion team, [2015-4-3] http://www.imt-2020.org.cn, (2014).

[3] L. Zhanjun, Z. Zhichao, Z. Shiyan and Z. Zhufan, "Cooperative RRU selection algorithm for multicast service in C-RAN wireless networks", Journal of Xidian University, vol. 6, no. 40, (2013).

[4] DTmobile innovation center. Evolution integration and innovation of 5G white paper [EB/OL]. [2014-17]. http://www.Datanggroup. cn /file /5gbps-ch.pdf, (2013).

[5] L. Zhanjun, C. Linglong, S. Yue, L. Zhongqi and C. Qianbin, "Packet scheduling based on the mechanism for direct communication in C-RAN wireless network", Journal of Chongqing University of posts and telecommunications, vol. 6, no. 40, (2013).

[6] Y. Li, D. Jin, J. Yuan and Z. Han, "Coalitional Games for Resource Allocation in the Device-to-Device Uplink Under laying Cellular Networks”, IEEE Transactions Wireless Communications, vol. 7, no. 13, (2014).

[7] L. Song, D. Niyato, Z. Han and E. Hossain, "Game-Theoretic resource allocation methods for device to device communication", IEEE Wireless Communications, vol. 3, no. 21, (2014)

[8] D. H. Le, K. W. Choi, W. S. Jeon and D. G. Jeong, "Resource Allocation Scheme for Device-to-Device Communication for Maximizing Spatial Reuse", IEEE Wireless Communications and Networking Conference, Shanghai, China, (2013).

[9] N. Forouzan and S. A. Ghorashi, "Inter-cell interference coordination in downlink orthogonal frequency division multiple access systems using Hungarian method", Institution of Engineering and Technology Communication, vol. 1, no. 7, (2013).

[10] S. Hakola, T. Chen, J. Lehtomaki and T. Koskela, "Device-To-Device (D2D) Communication in Cellular Network-Performance Analysis of Optimum and Practical Communication Mode Selection", IEEE Wireless Communications and Networking Conference, Sydney, Australia, (2010).

[11] Y. Ronlad, Z. Tao, J. Zhang and C. C. J. Kuo, "Muiticell OFDMA downlink resource allocation using a graphic framework", IEEE Transactions Vehicular Technology, vol. 7, no. 58, (2009). 


\section{Authors}

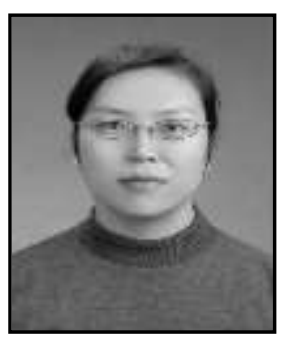

Hou Weina, was born in Hebei China in 1976, received her Bachelor degree form Hebei Normal University in 2000, received her Master degree from China University of Petroleum in 2003 and now she is an associate professor in Chongqing University of Posts and Telecommunications. Her interesting study field is antenna and demand propagation, wireless communication

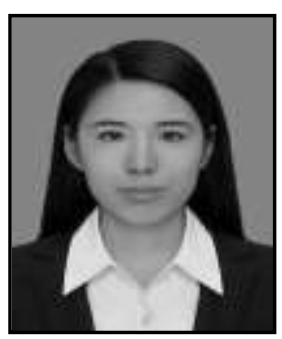

Yu Zhonghua, was born in Hei Longjiang Province in China in 1990. She received her bachelor degree in Chongqing University of Posts and Telecommunications in 2014, and now pursuing the master degree in the field of Telecommunication and Engineering in Chongqing University of posts and Telecommunications. Her interesting study field is wireless communication networks.

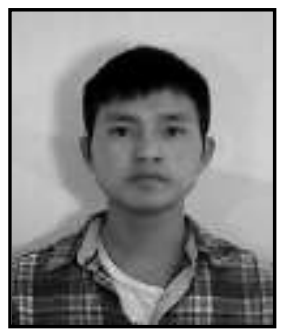

Chen Linglong, was born in Chognqing China in 1989, he received his bachelor degree in Chongqing University of Posts and Telecommunications in 2013, got his master degree in 2015 from Chongqing University of posts and Telecommunications. His interesting study field is wireless communication networks.

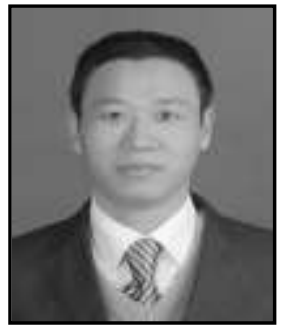

Liu Zhanjun, was born in Hebei China in 1975, received his Bachelor degree form Hebei Normal University in 2001, and got his Master degree form Chongqing University of Posts and Telecommunications Chongqing University of Posts and Telecommunications. His interesting study field is wireless communication networks, mobile telecommunication. 
International Journal of Multimedia and Ubiquitous Engineering

Vol.12, No.1 (2017) 